\title{
(Re)produire la ville à l'heure néolibérale : À la recherche de la valeur urbaine
}

Comparaison croisée de quatre anciens quartiers industriels Français (Caen, Le Havre) et Allemands (Leipzig, Dresde)

\section{Antonin Girardin}

\section{(2) OpenEdition}

Journals

Édition électronique

URL : https://journals.openedition.org/cdg/7539

DOI : $10.4000 /$ cdg. 7539

ISSN : 2107-7266

Éditeur

UMR 245 - CESSMA

Référence électronique

Antonin Girardin, «(Re)produire la ville à l'heure néolibérale : À la recherche de la valeur urbaine », Carnets de géographes [En ligne], 15 | 2021, mis en ligne le 30 avril 2021, consulté le 28 mai 2021. URL: http://journals.openedition.org/cdg/7539; DOI : https://doi.org/10.4000/cdg.7539

Ce document a été généré automatiquement le 28 mai 2021.

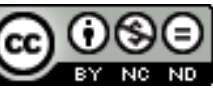

La revue Carnets de géographes est mise à disposition selon les termes de la Licence Creative Commons Attribution - Pas d'Utilisation Commerciale - Pas de Modification 4.0 International. 


\title{
(Re)produire la ville à l'heure néolibérale : À la recherche de la valeur urbaine
}

\author{
Comparaison croisée de quatre anciens quartiers industriels Français \\ (Caen, Le Havre) et Allemands (Leipzig, Dresde)
}

\section{Antonin Girardin}

1 Ces quarante dernières années sont marquées par le triomphe de la doctrine politique et économique néolibérale. Si les mécanismes globaux de dérégulation des marchés et de recomposition des compromis sociaux qu'elle induit sont bien connus, la faible proportion de travaux intégrant explicitement le rôle des transformations néolibérales du capitalisme sur les mutations urbaines témoigne du fait que la géographie urbaine peine encore à en identifier et à en qualifier l'importance sur la production locale de la ville, particulièrement en France où la tradition planificatrice et la puissance publique restent fortes. Concomitante à l'émergence de l'urbanisme par projet et à la diffusion, dans une grande diversité de contextes locaux, de modèles urbains génériques comme la « ville durable » ou la « ville créative », la néolibéralisation pose pourtant la question d'une transformation radicale du système de production de la ville. Celle-ci ne touche pas uniquement les politiques d'aménagement, mais concerne une grande partie des normes et des valeurs qui président la conception, les représentations et les appropriations de la ville contemporaine, c'est-à-dire des principes qui servent de guide à l'évaluation et à la légitimation des politiques urbaines et des compromis de classe qui les permettent.

2 Analysant ces transformations locales dans quatre anciens quartiers industriels de villes moyennes françaises et allemandes (Caen, Le Havre, Leipzig et Dresde) qui ont connu d'intenses processus de renouvellement urbain depuis près de trente ans, ce travail interroge les processus de revalorisation urbaine dans des espaces fortement dévalorisés au début des années 1990. La profondeur historique de l'analyse (1990-2020) vise à montrer que cette revalorisation prend la forme d'un processus de recapitalisation de l'espace qui articule valeurs symboliques (réputation, valeurs 
affectives attachées aux lieux) et valeur marchande (prix du foncier et de l'immobilier, attractivité économique, touristique etc.), selon un cycle précis de subordination des premières à la seconde, qui apparait typique du processus de la destruction/créatrice néolibérale. C'est dans ce cycle que se cimente un nouveau compromis de classe entre la bourgeoisie immobilière et certaines fractions de la classe d'encadrement capitaliste (Bihr, 1989) fortement dotées en capital culturel.

La revalorisation des quatre quartiers étudiés dans cette thèse (La Presqu'île de Caen, le quartier Saint-Nicolas au Havre, Plagwitz à Leipzig et Friedrichstadt à Dresde) s'organise autour de la spatialisation successive de différents types de capitaux qui ont pour effet de créer plusieurs formes de valeurs (valeurs d'usages, valeurs symboliques), dont le résultat est de générer les conditions de la valorisation économique. Le découpage de notre analyse autour du tryptique lefebvrien (2000) des dimensions de l'espace social (conception, représentation, appropriation) permet d'interroger ces dimensions les unes après les autres et d'aboutir à des modèles synthétiques de l'articulation de ces différents types de capitaux pour chacune des dimensions de l'espace social.

FIgURE 1. LES DIFFÉRENTES DIMENSIONS LOCALES DU PROCESSUS DE DESTRUCTION/CRÉATRICE DANS LES ANCIENS QUARTIERS INDUSTRIELS EN RECONVERSION

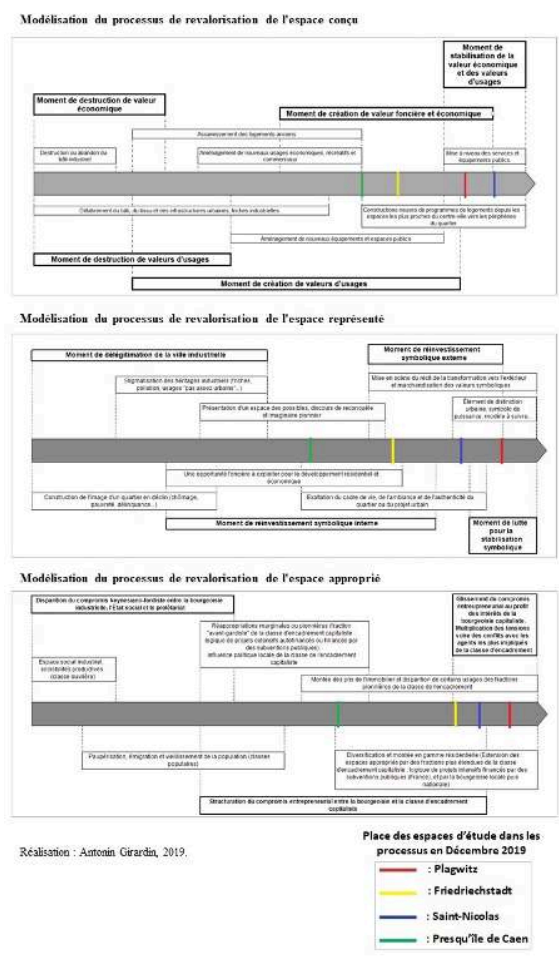

4 La revalorisation de l'ensemble des dimensions de l'espace social s'organise donc de manière similaire dans les quatre cas. Elle marque la transition d'espaces tournés vers la production industrielle vers des quartiers qui privilégient l'économie résidentielle ou récréative et qui ont pour rôle de mettre en scène une ville qui se renouvelle et se conforme aux modèles idéologiques dominants des villes tournées vers l'économie tertiaire et touristique, tout en mettant à distance les activités traditionnelles de production. Ces quatre cas illustrent un processus de génération progressive des conditions de comblement du Rent Gap (Smith, 1987), c'est-à-dire de la génération d'une 
demande résidentielle suffisante pour rentabiliser le comblement des friches industrielles par la production de logements.

Notre travail opère ainsi une comparaison processuelle assurée par le prisme de questionnements et de méthodes communes à chacun des quatre études de cas dans le but de produire une analyse fine d'échelle micro (celle du quartier) d'un processus commun dans des espaces marqués par des trajectoires historiques différentes. S'inscrivant dans le champ de la géographie critique, il vient ainsi renforcer les analyses du néolibéralisme en contribuant à combler un angle mort des théories néomarxistes anglo-saxonnes, souvent concentrées sur des échelles macro.

La modélisation des processus locaux de valorisation vient préciser la théorisation de la destruction/créatrice néolibérale (Théodore et Brenner, 2002, Peck et Tickell, 2002, etc.), en la rendant applicable à l'ensemble des anciens quartiers industriels en transformation. Il permet de montrer que le processus de néolibéralisation n'est pas uniquement un régime d'organisation macro-économique, mais qu'il se décline aussi localement à travers des formes spécifiques de production et de normalisation de la valeur urbaine Celles-ci procèdent d'une dialectique permanente entre évolutions structurelles de l'économie urbaine à l'échelle globale - qui déterminent une tendance aux processus de valorisation - et configurations localisées (rapport de force entre les classes, diversités des ressources symboliques disponibles, etc.).

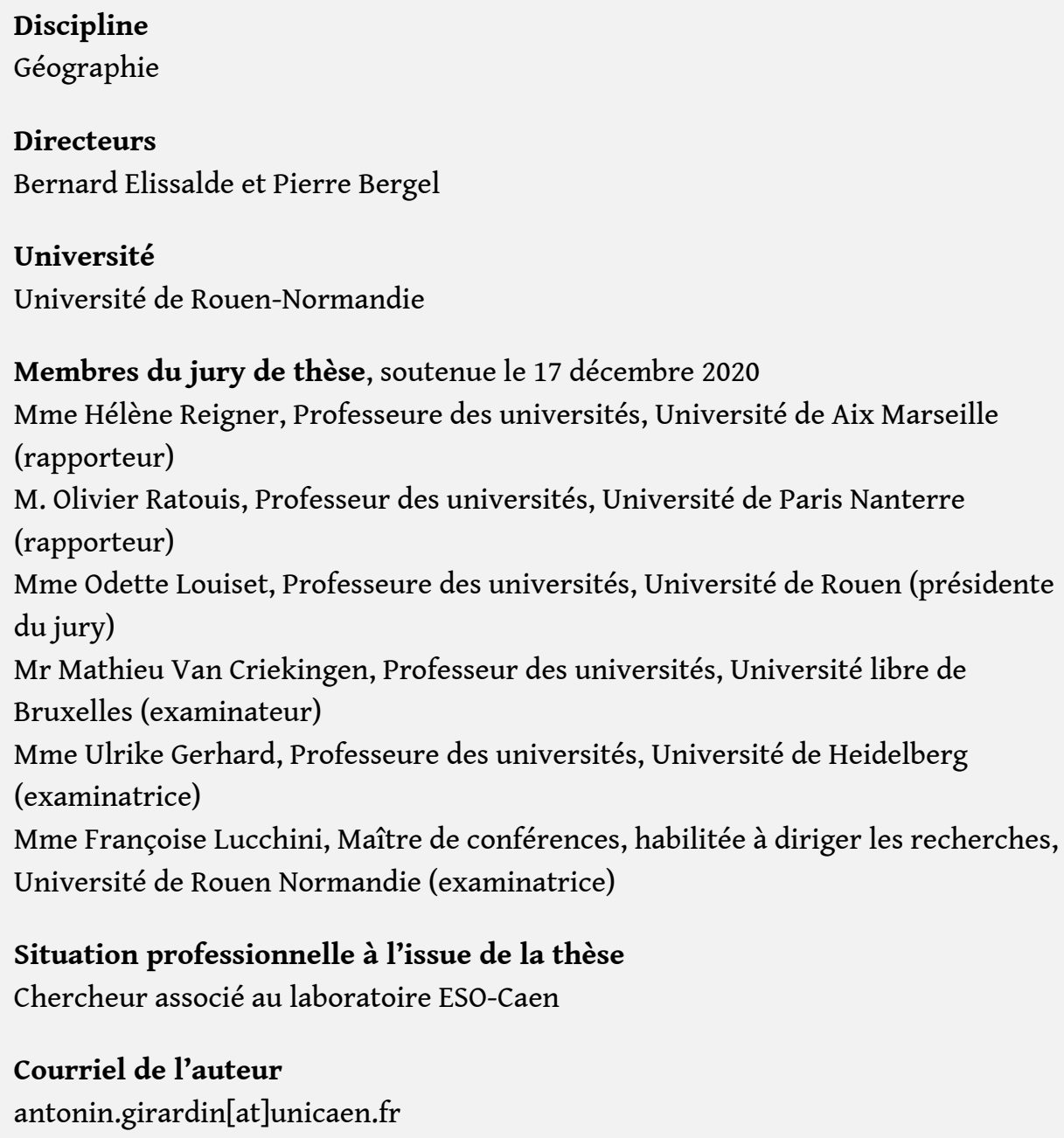




\section{BIBLIOGRAPHIE}

Bihr A. (1989), Entre bourgeoisie et prolétariat : l'encadrement capitaliste, Paris, L'Harmattan.

Brenner N., Theodore N. (2002), « Cities and the Geographies of “Actually Existing Neoliberalism" ", Antipode, Vol. 34, no. 3, p. 349-79. https://doi.org/10.1111/1467-8330.00246

Lefebvre H. (2000), La production de l'espace, Paris. Anthropos.

Peck J., Tickell A. (2002), « Neoliberalizing Space », Antipode, Vol. 34, no. 3, p. 380-404. https:// doi.org/10.1111/1467-8330.00247.

Smith N. (1987), « Gentrification and the rent gap », Annals of the Association of American Geographers, Vol. 77, no. 3, p. 462-465

INDEX

Thèmes : Carnets de soutenances

\section{AUTEUR}

\section{ANTONIN GIRARDIN}

Université de Rouen-Normandie, Université de Caen-Normandie antonin.girardin[at]unicaen.fr 